CrossMark \& click for updates

Cite this: J. Mater. Chem. C, 2016, 4, 2901

Received 13th October 2015, Accepted 18th November 2015

DOI: $10.1039 / \mathrm{c} 5 t \mathrm{tc} 03299 \mathrm{~g}$

www.rsc.org/MaterialsC

\section{Triphenylamine-functionalized tetraphenylpyrazine: facile preparation and multifaceted functionalities $\dagger$}

\author{
Ming Chen, ${ }^{a}$ Han Nie, ${ }^{b}$ Bo Song, ${ }^{b}$ Lingzhi Li, ${ }^{a}$ Jing Zhi Sun, ${ }^{a}$ Anjun Qin*ab and \\ Ben Zhong Tang*abc
}

\begin{abstract}
Aggregation-induced emission (AIE) is a unique photo-physical phenomenon and has become an emerging and hot research area. With the enthusiastic efforts paid by researchers, hundreds of AIE-active luminogens (AlEgens) have been generated but heterocyclic AlEgens are rarely reported. Recently, we enriched the family of AIEgens and reported a pyrazine-based AlEgen of tetraphenylpyrazine (TPP), which could be facilely functionalized by a post-synthetic strategy. In this work, we further expanded the TPP-based AIE system by covalently attaching one, two or four electron-donating triphenylamine moieties to the TPP core via Suzuki coupling, and TPP-TPA, TPP-2TPA and TPP-4TPA were produced, respectively. Thanks to their donor- $\pi$-acceptor structures, these luminogens exhibit multifunctional properties, such as excellent thermal stability (up to $504{ }^{\circ} \mathrm{C}$ ), large molar absorptivity, bright emission in the solid state (quantum yields up to 35.2\%), solvatochromism, and high two-photon absorption cross-sections (up to 480 GM). Furthermore, using TPP-TPA as the emitting layer, a triplelayer device was fabricated and a turn-on voltage, maximum luminance, current efficiency, power efficiency, and external quantum efficiency of $3.7 \mathrm{~V}, 17459 \mathrm{~cd} \mathrm{~m}^{-2}, 5.49 \mathrm{~cd} \mathrm{~A}^{-1}, 3.18 \mathrm{~lm} \mathrm{~W}^{-1}$ and $2.88 \%$ were realized, respectively. These results indicate a huge potential to develop high-tech applications based on these TPP-based AlEgens.
\end{abstract}

\section{Introduction}

Over the past decades, organic fluorophores have drawn much attention especially in optoelectronic and biological fields, due to their high emission efficiency, tuneable emission colour, facile post-functionalization and low toxicity. ${ }^{1}$ However, traditional fluorophores generally suffer from the aggregation-caused quenching (ACQ) effect: they are highly emissive in dilute

\footnotetext{
${ }^{a}$ MOE Key Laboratory of Macromolecular Synthesis and Functionalization, Department of Polymer Science and Engineering, Zhejiang University, Hangzhou 310027, China. E-mail: qinaj@zju.edu.cn,msqinaj@scut.edu.cn

${ }^{b}$ Guangdong Innovative Research Team, State Key Laboratory of Luminescent Materials and Devices, South China University of Technology, Guangzhou 510640, China

${ }^{c}$ Department of Chemistry, Institute for Advanced Study, Institute of Molecular Functional Materials, Hong Kong Branch of Chinese National Engineering Research Center for Tissue Restoration and Reconstruction, and State Key Laboratory of Molecular Neuroscience, The Hong Kong University of Science \& Technology, Clear Water Bay, Kowloon, Hong Kong, China.

E-mail: tangbenz@ust.hk

$\dagger$ Electronic supplementary information (ESI) available: ${ }^{1} \mathrm{H}$ and ${ }^{13} \mathrm{C}$ NMR spectra, HRMS spectra, absorption and photoluminescence spectra, CV curves, TGA and DSC curves. CCDC 1419384. For ESI and crystallographic data in CIF or other electronic format see DOI: 10.1039/c5tc03299g
}

solution but become weakly or non-emissive in high concentration or condensed phases like aggregate or solid state due to their planar conjugated structures. The ACQ effect has reduced the performance of traditional fluorophores when used in optoelectronic devices and as bio-probes. ${ }^{2}$ To alleviate this negative effect and to obstruct the formation of excimers or exciplexes upon aggregation, various approaches, such as physical doping or chemical modification with bulky groups, have been adopted. ${ }^{3}$ Nevertheless, the difficulties for precise control of doping concentration and avoidance of phase separation between the host and the guest during usage could not be ignored, whereas, in the latter case, tedious synthetic procedures and a lengthened operating time are also involved. In other words, these methods met with limited success.

One possible permanent strategy to solve this difficulty is to enable the intrinsic aggregation process of fluorophores to play positive roles in enhancing emission. In 2001, we indeed observed such a unique phenomenon: multiple phenyl-substituted silole derivatives are non-emissive when molecularly dissolved but can be induced to emit intensely upon aggregation. Since the emission was induced by aggregation, we thus defined it as "aggregation-induced emission (AIE)". ${ }^{4}$ Propeller-shaped AIE luminogens (AIEgens) drive them to dissipate the excited 
state energy via intramolecular rotation of their peripheral rotators in dilute solution, whereas, in the aggregate state this rotation is greatly restricted and the radiative transition channel is opened up. Accordingly, a mechanism of the restriction of intramolecular rotation (RIR) was proposed and has thoroughly been proved by plenty of experimental and theoretical studies. ${ }^{5}$

Excited by the AIE concept and enlightened by the RIR mechanism, researchers have designed and synthesized hundreds of AIEgens especially based on the cores of silole, ${ }^{6}$ tetraphenylethene (TPE), ${ }^{7}$ distyrylanthracene (DSA), ${ }^{8}$ and tetraphenyl-1,4-butadiene (TPBD) ${ }^{9}$. However, heterocycle-based AIE cores with diverse electronic properties are still rare but in great demand because they could further expand the functionalities and promote high-tech applications in organic light-emitting diodes (OLEDs), fluorescence sensors, biological imaging, and so on. ${ }^{10}$ Very recently, we successfully generated a new type of such AIEgen of tetraphenylpyrazine (TPP), which possesses lots of charming advantages, such as easy preparation, facile modification, excellent stability, tuneable emission colour and definite luminescence mechanism. ${ }^{11}$ It is thus envisioned that TPP could also act as another powerful AIE core, and attract considerable interest in materials and biological sciences.

To demonstrate its versatility as an AIE core, in this paper, we further expanded its family by covalently bonding with the triphenylamine (TPA) moiety, which is well known for its luminescence properties, electron-donating ability, and holetransporting capability. ${ }^{12}$ However, most TPA derivatives suffer from the ACQ effect. A powerful strategy to enable the TPA moiety to increase the emission efficiency in the solid state is to covalently bind it to an AIE core, like TPE. ${ }^{13}$ More importantly, the remaining charge-transporting ability makes the newly generated luminogens perform much better when used in OLEDs. In addition, thanks to its electron-donating properties, molecules containing TPA units and electron-withdrawing groups are always in association with large two-photon absorption cross-sections, which is exceedingly desirable for bioimaging and photodynamic therapy applications. ${ }^{14}$ Indeed, three TPP-based molecules of TPP-TPA, TPP-2TPA, and TPP4TPA, in which one, two and four TPA moieties are covalently attached to the TPP core, show multi-functional properties and are potentially applicable in OLEDs, two-photon absorption materials, etc.

\section{Results and discussion}

\section{Synthesis}

To obtain TPP-based luminogens with flexible structures, the intermediates of Br-substituted TPPs are indispensable. Besides TPP-2Br (the structure is shown in Scheme 1), which has been reported in our recent work, ${ }^{11}$ another two intermediates of TPP-Br and TPP-4Br (Scheme 1, their synthetic procedures are given in the $\mathrm{ESI} \dagger$ ) were prepared in a facile approach in this paper. ${ }^{15,16}$ The TPP-based luminogens were

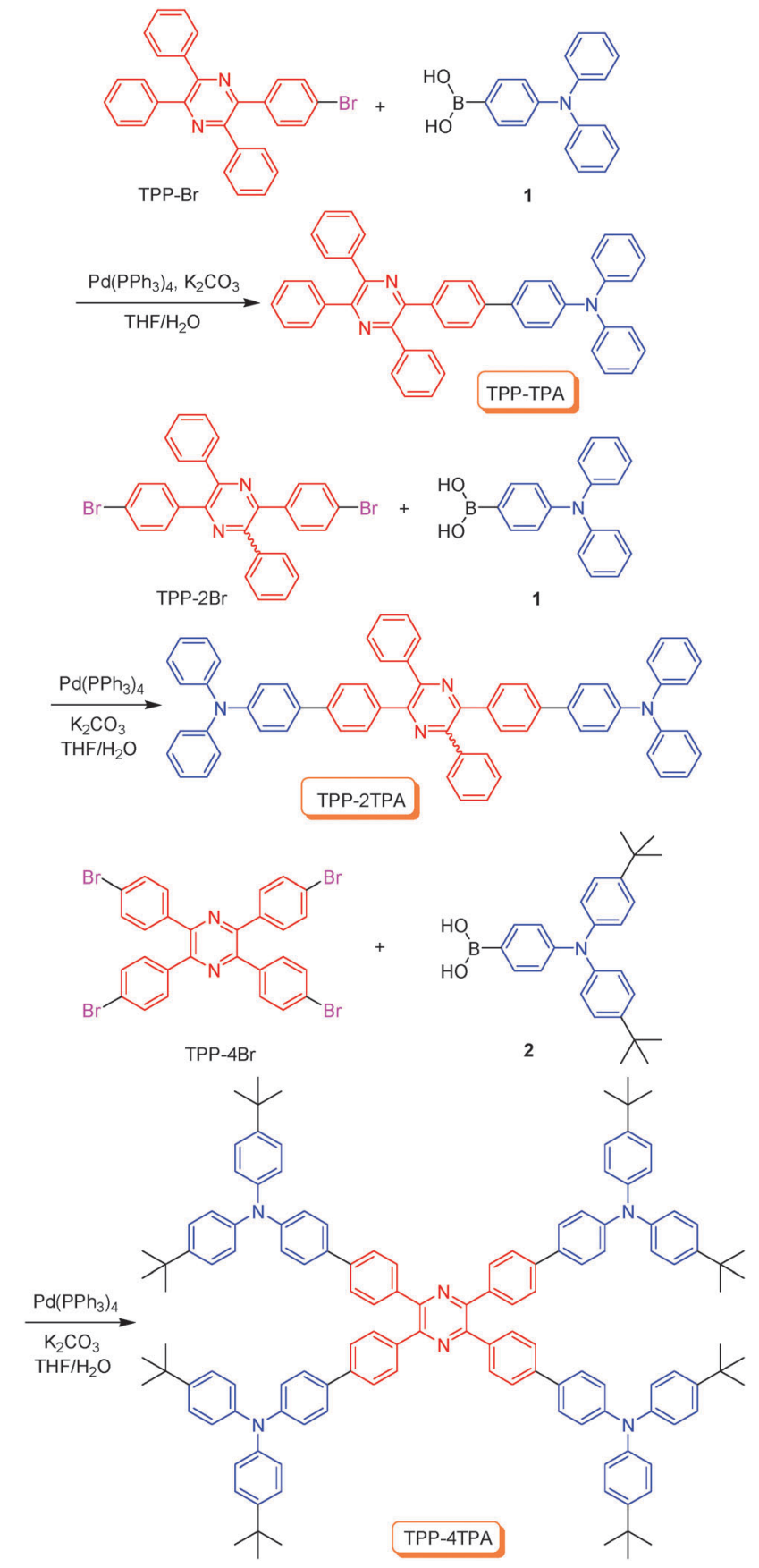

Scheme 1 Synthetic routes to TPP-TPA, TPP-2TPA and TPP-4TPA.

then prepared by classical Suzuki coupling. Among which, TPP-TPA and TPP-2TPA were obtained by the reaction of TPP-Br and TPP-2Br with commercially available 4-(diphenylamino)-phenylboronic acid (1) in moderate yields, respectively. However, the product yielded from TPP-4Br and 1 was insoluble probably due to its rigidity. Delightfully, TPP-4TPA was successfully obtained by the reaction of TPP- $4 \mathrm{Br}$ and isobutyl-decorated 1 , that is, compound $2 .^{17}$ TPP-TPA, TPP-2TPA, TPP-4TPA and their intermediates were fully characterized by spectroscopy techniques and satisfactory analysis data corresponding to their structures were obtained (Fig. S1-S20, ESI $\dagger$ ). 


\section{Thermal stability}

After confirming their structures, we investigated the thermal stability of the obtained luminogens by thermogravimetric analysis (TGA) with a heating rate of $10{ }^{\circ} \mathrm{C} \mathrm{min}^{-1}$ under nitrogen (Fig. S21, ESI $\dagger$ ). The temperatures for $5 \%$ weight loss $\left(T_{\mathrm{d}}\right)$ of TPP-TPA and TPP-2TPA were recorded to be as high as 402 and $504{ }^{\circ} \mathrm{C}$, respectively, due to their fully conjugated aromatic structures. It is worth noting that these values are higher than those of TPE-TPA and TPE-2TPA, which were reported to be 360 and $430{ }^{\circ} \mathrm{C}$, respectively, ${ }^{18}$ further confirming that the TPP core possesses high thermal stability. Unexpectedly, TPP-4TPA began to decompose at $171{ }^{\circ} \mathrm{C}$, followed by a second decomposition at a temperature of over $500{ }^{\circ} \mathrm{C}$. After a careful inspection, we ascribe the first weight loss of TPP-4TPA to the isobutyl groups on its periphery, whereas the central aromatic moieties remain intact until the temperature reaches $500{ }^{\circ} \mathrm{C}$. The high thermal stability of the TPP-based luminogens potentially enables them to find broad applications.

\section{Photo-physical properties}

We first investigated the absorption properties of these luminogens in dilute THF solutions. As shown in Fig. 1, with an increase in the numbers of TPA moieties, the maximum absorption $\left(\lambda_{\mathrm{ab}}\right)$ of TPP-TPA, TPP-2TPA and TPP-4TPA red-shifted from 363 to $387 \mathrm{~nm}$, suggesting that the molecular conjugation could be slightly influenced by increasing the electron-donating groups. In contrast, the molar absorptivity of TPP-TPA, TPP-2PTA and TPP-4TPA enhanced linearly and is nearly proportional to the attached numbers of TPA moieties. This phenomenon could be attributed to the multiple intramolecular charge transfers between the TPA moieties to the TPP cores. The more acceptor (A) and donor (D) pairs there are, the higher the molar absorptivity that could be achieved.

TPP is AIE-active, are these TPP-based luminogens also AIE-active? To answer this question, we studied their photoluminescence (PL) spectra in THF/water mixtures with different water fractions. As shown in Fig. 2 and Fig. S22 (ESI†), TPP-TPA

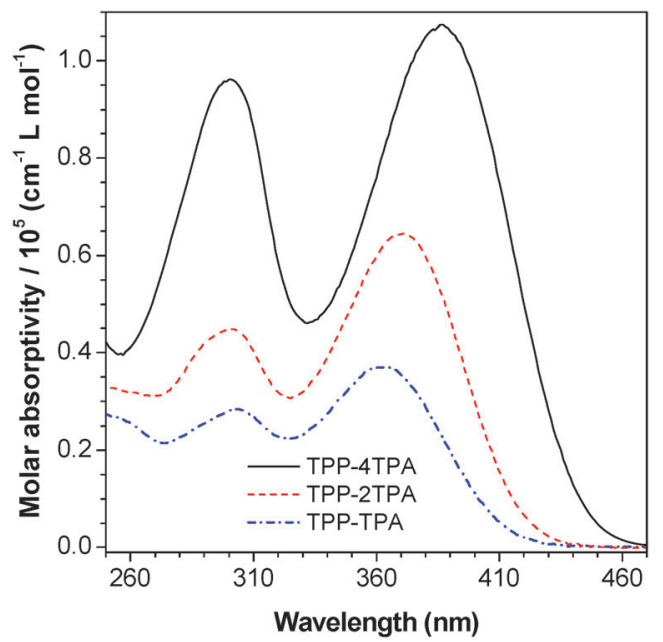

Fig. 1 UV-vis spectra of TPP-TPA, TPP-2TPA and TPP-4PTA in THF.
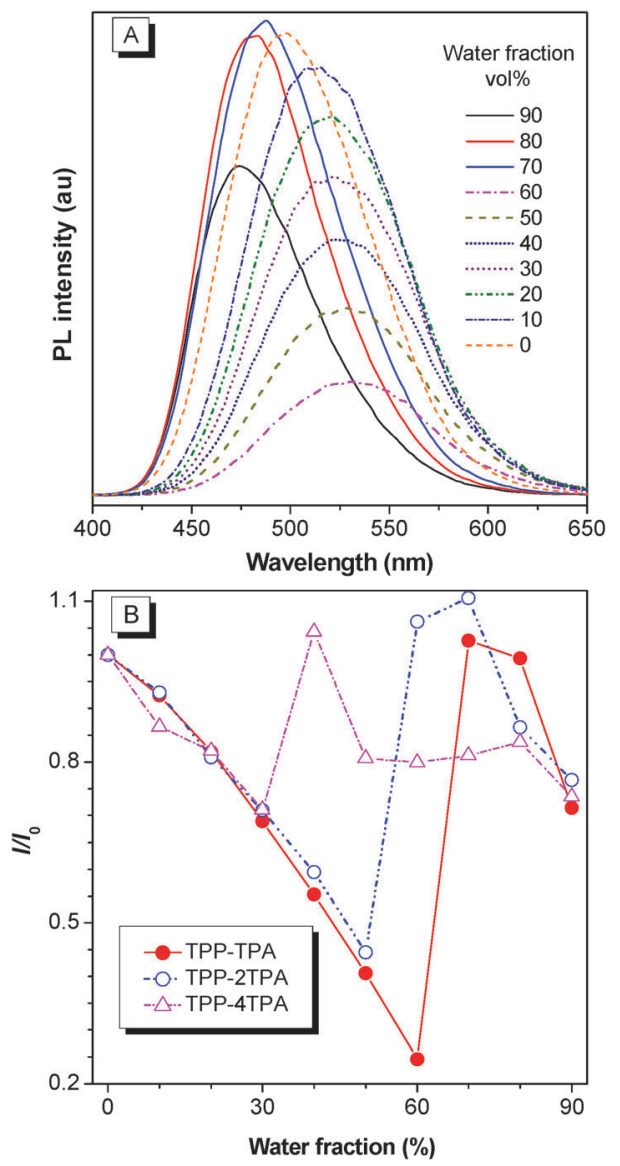

Fig. 2 (A) PL spectra of TPP-TPA in THF/water mixtures with different water fractions; $\lambda_{\mathrm{ex}}=363 \mathrm{~nm}$; concentration: $10 \mu \mathrm{M}$. (B) Changes of relative PL intensity of TPP-TPA, TPP-2TPA and TPP-4TPA in THF/water mixtures with different water fraction.

emits at $498 \mathrm{~nm}$ in dilute THF solution. After the addition of a poor solvent (i.e. water), the emission of the system weakened with a red-shift of their peaks at the same time. Specifically, more than $75 \%$ of its fluorescence intensity was quenched and a bathochromic shift of about $34 \mathrm{~nm}$ could be discerned at the water fraction $\left(f_{\mathrm{w}}\right)$ of $60 \%$. Afterwards, a remarkable fluorescence enhancement with a blue-shift of peaks was recorded. The decrease in fluorescence intensity and the red-shift of its peaks are due to the twisted intramolecular charge-transfer (TICT) effect of TPP-TPA in the gradually strengthened polar mixed solvent of $\mathrm{THF} /$ water with a higher fraction of polar water. ${ }^{19}$ Whereas, the enhanced emission and the blue-shifted peak could be attributed to the aggregate formation due to the worsened solvating power of the aqueous mixture and the lower impact of the polarity of solvent on TPP-TPA. Simultaneously, the rotation of the phenyl rings of TPP-TPA was greatly restricted in the aggregate state, and thus the unique aggregation-enhanced emission (AEE) occurred. The weaker emission of TPP-TPA in the THF/water mixtures with $f_{\mathrm{w}}$ of 80 and $90 \%$ compared with that with $f_{\mathrm{w}}$ of $70 \%$ is probably due to the difference in aggregate morphology. ${ }^{20}$

The similar D-A structures of TPP-2TPA and TPP-4TPA also enable them to have ACQ plus AEE effects dominated by the 
TICT and RIR, respectively (Fig. S23 and S24, ESI $\dagger$ ). Nevertheless, there are some evident differences between them (Fig. 2B). For example, from TPP-TPA and TPP-2TPA to TPP4TPA, the enhanced molecular rigidity has greatly decreased the solubility, and thus led to the formation of aggregates at a lower $f_{\mathrm{w}}$. In addition, the AEE effect was discounted obviously with the increase of TPA moieties because more TPA groups attached to the TPP core might generate a larger free volume, in which the rotation of the phenyl rings could not be restricted efficiently after aggregation. ${ }^{21}$ This explanation was proven by the absolute quantum yield $\left(\Phi_{\mathrm{F}}\right)$ measurement. The values of TPP-TPA, TPP-2PTA and TPP-4TPA in solid states were recorded to be $35.2,16.5$ and $12.4 \%$, respectively (Table S1, ESI $\dagger$ ).

\section{Solvatochromism}

The sensitivity of these TPP-based luminogens to polar solvents was further confirmed by measuring their photo-physical properties in various organic solvents with different polarities. The UV-vis spectra of TPP-TPA showed that its absorption profiles changed little and were nearly free from the influence of solvent polarity. Contrarily, distinct changes in emission colour were observed in these luminogens. For example, when TPP-TPA was dissolved in hexane, it emits a deep blue light at $441 \mathrm{~nm}$. However, further increase of solvent polarity remarkably red-shifted its emission. The bathochromic shift was identified to be as large as $108 \mathrm{~nm}$ in solvents from hexane to acetonitrile. Moreover, similar solvatochromism was also observed in TPP-2TPA and TPP-4TPA (Fig. 3 and Fig. S25 and $\mathrm{S} 26, \mathrm{ESI} \dagger)$.
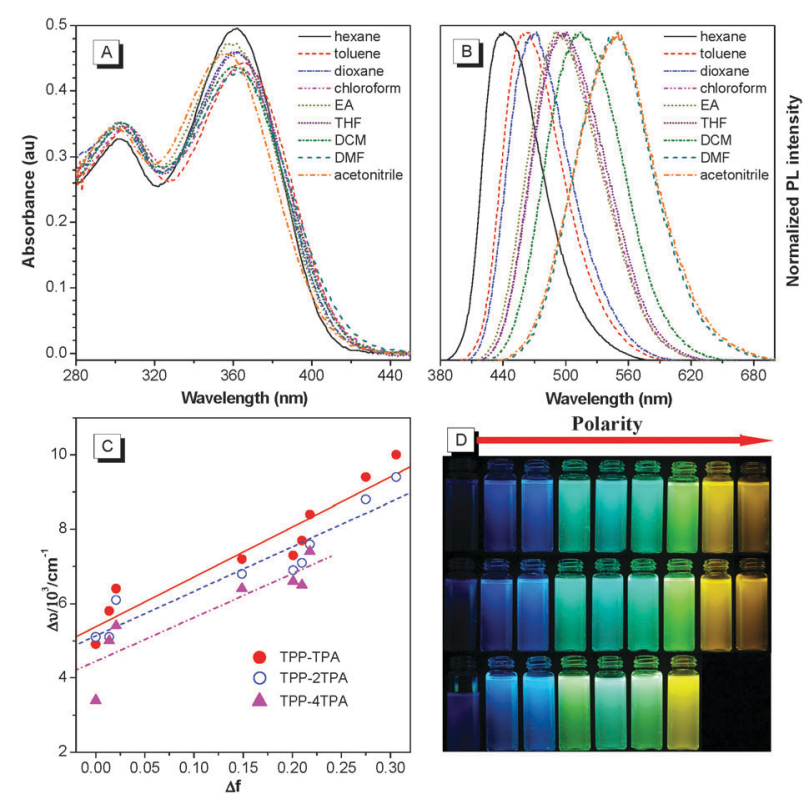

Fig. 3 UV-vis spectra (A) and normalized PL spectra (B) of TPP-TPA in solvents with various polar parameters. (C) Plot of Stokes shift $(\Delta v)$ of TPPTPA, TPP-2TPA and TPP-4TPA versus $\Delta f$ of their solutions. (D) Photographs of TPP-TPA, TPP-2TPA and TPP-4TPA (from top to bottom) in solvents with increased polar parameters (from left to right) taken under the irradiation of $365 \mathrm{~nm}$ UV light.
Subsequently, we quantitatively analysed the influence of solvent polarity, characterized by $\Delta f$, on the Stokes shift using the Lippert-Mataga equation: ${ }^{22}$

$$
\begin{gathered}
\Delta v \equiv v_{\mathrm{ab}}-v_{\mathrm{em}}=\frac{2 \Delta f}{h c a^{3}}\left(\mu_{\mathrm{e}}-\mu_{\mathrm{g}}\right)^{2}+\text { const. } \\
\Delta f=\frac{\varepsilon-1}{2 \varepsilon+1}-\frac{n^{2}-1}{2 n^{2}+1}
\end{gathered}
$$

where $\Delta v$ is the Stokes shift, $h$ is the Planck constant, $c$ is the speed of light, $a$ is the radius of the chromophore, $\mu_{\mathrm{e}}$ and $\mu_{\mathrm{g}}$ are the dipole moments in the excited (e) and ground (g) states, $\Delta f$ is the solvent polarity parameter, and $\varepsilon$ and $n$ are the dielectric constant and refractive index of the solvent, respectively. According to the plots of $\Delta v$ vs. $\Delta f$, TPP-TPA, TPP-2TPA and TPP-4TPA exhibit notable positive slopes after linear fitting, with their values deduced to be 13454, 12026 and 11827 , respectively (Fig. 3C). These results suggest that a very effective intramolecular charge transfer process could take place from the electron-donating TPA to the electron-withdrawing TPP units in these luminogens.

\section{Single crystal structure}

The single crystal structure and relevant packing information are crucial for further understanding the photo-physical behaviour of the molecules. Fortunately, a single crystal of TPP-TPA (CCDC 1419384) suitable for X-ray diffraction analysis was obtained by slow evaporation of the chloroform/methanol mixture. Fig. 4 shows the ORTEP drawing of TPP-TPA and the packing of adjacent molecules in the crystal cell. Owing to the respective propeller-like and cone-shaped TPP and TPA moieties, the whole molecule adopts a twisted conformation. Moreover, the molecules are loosely stacked in an antiparallel fashion, suggesting that TPP-TPA is a polar molecule. Meanwhile, this conformation also makes TPP-TPA easy to boost the emission in the aggregate state as the intramolecular motion is suppressed by the steric effect of adjacent molecules to open up the irradiative transition channel.

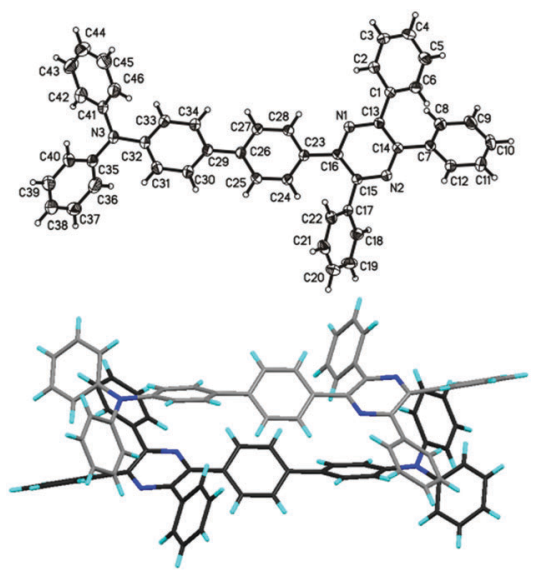

Fig. 4 ORTEP drawing of TPP-TPA and the packing of adjacent molecules in crystal cell. 
HOMO
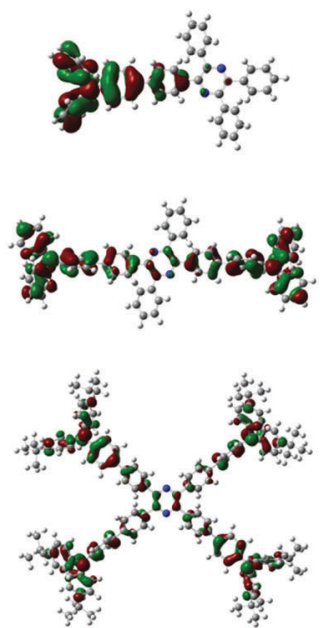

LUMO
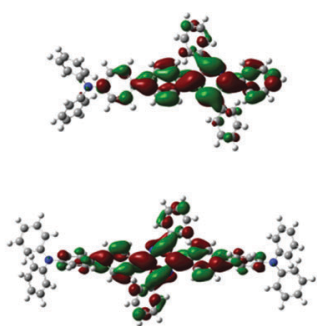

TPP-4TPA

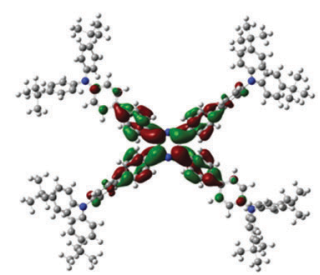

Fig. 5 Optimized molecular conformation and molecular orbital amplitude plots of HOMO and LUMO energy levels of TPP-TPA, TPP-2TPA and TPP4TPA. One of the two isomers of TPP-2TPA was used.

\section{Theoretical calculations}

To gain insight into the molecular structures of the TPP-based luminogens, theoretical calculations were performed. Density functional theory (DFT) with a B3LYP/6-31G (d) basis set was used and the results of the molecular energy levels are shown in Fig. 5 and Table S2 (ESI $\dagger$ ). We can see that the electron clouds of the HOMOs and the LUMOs of these luminogens are mainly located on the TPA and TPP groups, respectively, demonstrating a remarkable charge separation between the ground and excited states. From the calculation, we can also conclude that the more D-A pairs in the molecules, the more charge separation is involved, which further confirms our observation and explanation about the photo-physical properties of the TPP-based luminogens.

\section{Electrochemical properties}

Besides the theoretical evaluation, we also used a cyclic voltammogram (CV) technique to measure their energy levels experimentally. From the voltammogram curves shown in Fig. S27 (ESI $\dagger$ ), the oxidization peaks with onset potentials of TPP-TPA, TPP-2TPA and TPP-4TPA at 0.66, 0.57 and $0.47 \mathrm{eV}$, respectively, could be easily observed in the positive scan direction, which is responsive to electron-rich TPA moieties in these luminogens. The more TPA groups involved, the greater the potential of oxidation in these luminogens. We thus infer that the electronic properties of the ground state are mainly controlled by the TPA moieties in these luminogens.

From the CV measurements, the HOMO energy levels of TPPTPA, TPP-2TPA and TPP-4TPA were calculated to be $-5.04,-4.95$ and $-4.85 \mathrm{eV}$, respectively. Such high values are comparable to typical TPA derivatives and endow them with hole-transporting ability. By subtracting the optical band gap energies (estimated from the absorption spectra) from the HOMO energy levels, all the LUMOs of them were deduced to be $c a .2 .0 \mathrm{eV}$, which are much higher than that of TPP $(-2.58 \mathrm{eV})$ (Table S2, ESI $\dagger$ ).

\section{Two-photon absorption}

Molecules with D- $\pi$-A structures usually exhibit large twophoton absorption cross-sections $\left(\sigma_{\mathrm{TPA}}\right)$. We thus measured these values by employing a two-photon excited fluorescence technique in the wavelength range of 700-900 $\mathrm{nm}$. The results show that TPP-TPA, TPP-2TPA and TPP-4TPA emit with peaks at 532, 535 and $561 \mathrm{~nm}$, respectively, upon exciting by a laser in the wavelength range of 700-900 $\mathrm{nm}$. These peaks are about $20 \mathrm{~nm}$ red-shifted compared to the one-photon excited fluorescence, which is probably caused by the system errors of varying apparatus (Fig. S28, ESI $\dagger$ ). Moreover, another important value of $\sigma_{\text {TPA }}$ was also calculated. From Fig. 6 , we can see that the $\sigma_{\text {TPA }}$ values of the TPP-based luminogens changed with the molecular structures as well as the excitation wavelengths. Furthermore, both TPP-TPA and TPP-2TPA possess similar $\sigma_{\text {TPA }}$ values, whereas that of TPP-4TPA is almost twice as those of TPP-TPA and TPP-2PTA, and the highest value of $480 \mathrm{GM}$ was deduced. In addition, by choosing $\sigma_{\text {TPA }}$ at $800 \mathrm{~nm}$ as a criterion, which is a mature and most used laser source for biological imaging, the values of TPP-TPA, TPP-2TPA and TPP-4TPA were found to be 80,51 , an $195 \mathrm{GM}$, respectively. It is worthy of note that these values are still superior to those of coumarin and fluorescein, whose values are generally in the range of $36-45 \mathrm{GM}^{23}$

\section{Electroluminescence}

Thanks to the high emission of TPP-TPA in the solid state, we used it as an emitting layer to fabricate OLEDs. A typical triplelayer device with a configuration of ITO/NPB (60 nm)/TPP-TPA $(20 \mathrm{~nm}) / \mathrm{TPBi}(40 \mathrm{~nm}) / \mathrm{LiF}(1 \mathrm{~nm}) / \mathrm{Al}$ (Device I) was fabricated, in which NPB [ $N, N^{\prime}$-di(1-naphthyl)- $N, N^{\prime}$-diphenyl-benzidine] functions as the hole-transporting layer and TPBi [1,3,5-tri(1-phenyl$1 H$-benzo[ $d]$ imidazol-2-yl)phenyl] acts as the electron-transporting layer. The results show that device I emits sky blue light at $482 \mathrm{~nm}$ with its EL spectrum resembling the PL spectrum of TPP-TPA in solid film, indicating that the charges could transport in balance and excitations were successfully combined in the emitting layer (Fig. 7A). Moreover, the device also performed

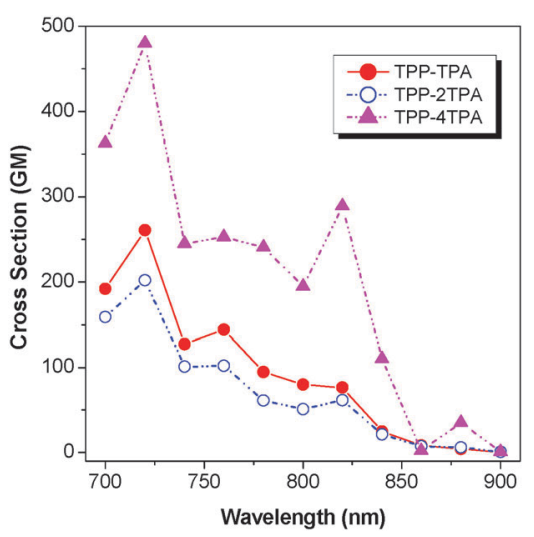

Fig. 6 The plots of two-photon absorption cross-section $\left(\sigma_{\text {TPA }}\right)$ of TPPTPA, TPP-2PTA and TPP-4TPA vs. excitation wavelength in dichloromethane. The concentrations of TPP-TPA, TPP-2TPA, TPP-4TPA are $6.37,5.28$ and $2.77 \mu \mathrm{M}$, respectively. 

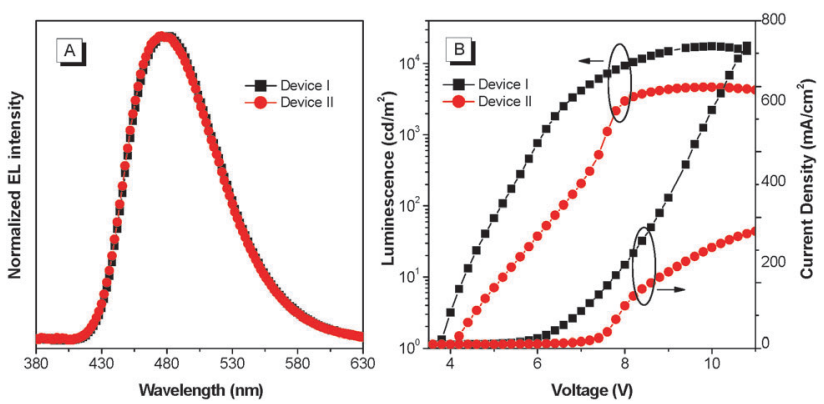

Fig. 7 (A) EL spectra of devices I and II using TPP-TPA as emitting layers (B) Luminescence and current density versus voltage characteristics of TPP-TPA based devices.

Table 1 EL performance of devices based on TPP-TPA

\begin{tabular}{lllllll}
\hline & $\begin{array}{l}\lambda_{\mathrm{EL}} \\
(\mathrm{nm})\end{array}$ & $\begin{array}{l}V_{\mathrm{On}}{ }^{b} \\
(\mathrm{~V})\end{array}$ & $\begin{array}{l}L_{\max }{ }^{a} \\
\left(\mathrm{~cd} \mathrm{~m}^{-2}\right)\end{array}$ & $\begin{array}{l}\eta_{\mathrm{C}}{ }^{a} \\
\left(\mathrm{~cd} \mathrm{~A}^{-1}\right)\end{array}$ & $\begin{array}{l}\eta_{\mathrm{P}}{ }^{a} \\
\left(\mathrm{~lm} \mathrm{~W}^{-1}\right)\end{array}$ & $\begin{array}{l}\mathrm{EQE}^{a} \\
(\%)\end{array}$ \\
\hline I & 482 & 3.7 & 17459 & 5.49 & 3.18 & 2.88 \\
II & 476 & 4.0 & 4692 & 4.72 & 3.53 & 2.52
\end{tabular}

${ }^{a}$ The maximum luminescence $\left(L_{\max }\right)$, current efficiency $\left(\eta_{\mathrm{C}}\right)$, power efficiency $\left(\eta_{\mathrm{P}}\right)$ and external quantum efficiency are the maximum values for the devices. ${ }^{b} V_{\text {on }}$ is the turn-on voltage at $1 \mathrm{~cd} \mathrm{~m}^{-2}$.

well with a turn-on voltage, maximum luminance, current efficiency, power efficiency, and external quantum efficiency of $3.7 \mathrm{~V}, 17459 \mathrm{~cd} \mathrm{~m}^{-2}, 5.49 \mathrm{~cd} \mathrm{~A}^{-1}, 3.18 \mathrm{~lm} \mathrm{~W}^{-1}$ and $2.88 \%$, respectively, which are almost identical to the values for multilayer devices that used TPE-TPA as the emitting layer (Fig. 7B and Table 1$).{ }^{18}$

TPP-TPA possesses a high-lying HOMO energy level, which is anticipated to possess good hole-transporting ability besides serving as an emitting layer. We thus simplified the device configuration by removing the HTL but thickening the emitting layer to $80 \mathrm{~nm}$ (Device II, Table 1). Unfortunately, the device performance is not as good as we expected although its EL spectrum is quite similar to that of device I, suggesting that other factors, such as molecular planarity and morphological homogeneity, might be involved in influencing the hole-transporting properties of TPP-TPA.

\section{Conclusions}

In this work, by covalently binding electron-donating TPA moieties to an electron-accepting TPP core via Suzuki coupling, three luminogens of TPP-TPA, TPP-2TPA and TPP-4TPA were successfully obtained. Thanks to their D- $\pi$-A structures, these luminogens exhibit multiple functional properties. They possess large molar absorptivity, show strong emission intensity in the solid state, demonstrate distinct solvatochromism, and exhibit high two-photon absorption cross-sections. Moreover, these luminogens are thermally stable because of their conjugated aromatic structures. Based on the high absolute quantum yield $(35.2 \%)$ of TPP-TPA in the solid state, a triplelayer device using it as EML was fabricated, which gave high performance with a low $V_{\text {on }}$ of $3.7 \mathrm{~V}$, high $L_{\max }$ of $17459 \mathrm{~cd} \mathrm{~m}^{-2}$ and a good EQE of $2.88 \%$. This work not only furnishes a promising platform for the preparation of TPP-based luminogens with mutable structures, high emission, and multifaceted functionalities, but also demonstrates the possibility of applying these AIEgens in high-tech areas.

\section{Acknowledgements}

This work was partially supported by the key project of the Ministry of Science and Technology of China (2013CB834702), the National Science Foundation of China (21490571, 21222402 and 21174120), and the Research Grants Council of Hong Kong (16301614) and the Innovation and Technology Commission (ITC-CNERC14S01). A. J. Q. and B. Z. T. thank the support from Guangdong Innovative Research Team Program (201101C0105067115) and the Fundamental Research Funds for the Central Universities (2015ZY013).

\section{Notes and references}

1 (a) C. H. Chien, C. K. Chen, F. M. Hsu, C. F. Shu, P. T. Chou and C. H. Lai, Adv. Funct. Mater., 2009, 19, 560; (b) L. Zöphel, V. Enkelmann and K. Müllen, Org. Lett., 2013, 15, 804; (c) J. P. Anderson, B. L. Reynolds, K. Baum and G. Williams, Nano Lett., 2010, 10, 788; (d) S. You, Q. Cai, Y. Zheng, B. He, J. Shen, W. Yang and M. Yin, ACS Appl. Mater. Interfaces, 2014, 6, 16327.

2 J. B. Birks, Photophysics of Aromatic Molecules, Wiley, London, 1970.

3 (a) S. A. Swanson, G. M. Wallraff, J. P. Chen, W. Zhang, L. D. Bozano, K. R. Cater, J. R. Salem, R. Villa and J. C. Scott, Chem. Mater., 2003, 15, 2305; (b) H. J. Cho, D. H. Hwang, J. I. Lee, Y. K. Jung, J. H. Park, J. Lee, S. K. Lee and H. K. Shim, Chem. Mater., 2006, 18, 3780; (c) K. Y. Pu, B. Zhang, Z. Ma, P. Wang, X. Y. Qi, R. F. Chen, L. H. Wang, Q. L. Fan and W. Huang, Polymer, 2006, 47, 1970; (d) S. Xiao, M. Nguyen, X. Gong, Y. Cao, H. Wu, D. Moses and A. J. Heeger, Adv. Funct. Mater., 2003, 13, 25; (e) Y. Zou, J. Zou, T. Ye, H. Li, C. Yang, H. Wu, D. Ma, J. Qin and Y. Cao, Adv. Funct. Mater., 2013, 23, 1781; $(f)$ Z. Li, B. Jia, Z. Wu, P. Liu, L. Ma, X. Lei, D. Wang, G. Zhou, H. Hu and X. Hou, J. Mater. Chem. C, 2013, 1, 2183.

4 J. Luo, Z. Xie, J. W. Y. Lam, L. Cheng, H. Chen, C. Qiu, H. S. Kwok, X. Zhan, Y. Liu, D. Zhu and B. Z. Tang, Chem. Commun., 2001, 1740.

5 (a) J. Chen, C. C. W. Law, J. W. Y. Lam, Y. Dong, S. M. F. Lo, I. D. Williams, D. Zhu and B. Z. Tang, Chem. Mater., 2003, 15, 1535; (b) Z. Li, Y. Dong, B. Mi, Y. Tang, M. Häussler, H. Tong, Y. Dong, J. W. Y. Lam, Y. Ren, H. H. Y. Sung, K. S. Wong, P. Gao, I. D. Williams, H. S. Kwok and B. Z. Tang, J. Phys. Chem. B, 2005, 109, 10061; (c) Y. Ren, J. W. Y. Lam, Y. Dong, B. Z. Tang and K. S. Wong, J. Phys. Chem. B, 2005, 109, 1135; (d) A. Qin, J. W. Y. Lam, F. Mahtab, C. K. W. Jim, L. Tang, J. Z. Sun, H. H. Y. Sung, I. D. Williams and B. Z. Tang, Appl. Phys. Lett., 2009, 94, 253308; (e) T. Zhang, Y. Jiang, Y. Niu, D. Wang, Q. Peng and 
Z. Shuai, J. Phys. Chem. A, 2014, 118, 9094; $(f)$ Q. Wu, Q. Peng, Y. Niu, X. Gao and B. Z. Tang, J. Phys. Chem. A, 2012, 116, 3881; $(g)$ S. L. Deng, T. L. Chen, W. L. Chien and J. L. Hong, J. Mater. Chem. C, 2014, 2, 651.

6 (a) Y. Dong, J. W. Y. Lam, A. Qin, Z. Li, J. Liu, J. Sun, Y. Dong and B. Z. Tang, Chem. Phys. Lett., 2007, 446, 124; (b) H. Shi, J. Liu, J. Geng, B. Z. Tang and B. Liu, J. Am. Chem. Soc., 2012, 134, 9569; (c) Z. Zhao, Z. Wang, P. Lu, C. Y. K. Chan, D. Liu, J. W. Y. Lam, H. H. Sung, I. D. Williams, Y. Ma and B. Z. Tang, Angew. Chem., Int. Ed., 2009, 48, 7608; (d) L. Chen, Y. B. Jiang, H. Nie, P. Lu, H. H. Y. Sung, I. D. Williams, H. S. Kwok, F. Huang, A. J. Qin, Z. J. Zhao and B. Z. Tang, Adv. Funct. Mater., 2014, 24, 3621; (e) R. E. Scalise, P. A. Caradonna, H. J. Tracy, J. L. Mullin and A. E. Keirstead, J. Inorg. Organomet. Polym. Mater., 2014, 24, 431; $(f)$ J. Mei, J. Wang, J. Z. Sun, H. Zhao, W. Z. Yuan, C. M. Deng, S. M. Chen, H. H. Y. Sung, P. Lu, A. J. Qin, H. S. kwok, Y. G. Ma, I. D. Williams and B. Z. Tang, Chem. Sci., 2012, 3, 549; (g) J. Liu, J. W. Y. Lam and B. Z. Tang, J. Inorg. Organomet. Polym. Mater., 2009, 19, 249; (h) Z. Zhao, B. He and B. Z. Tang, Chem. Sci., 2015, 6, 5347.

7 (a) Y. Dong, J. W. Y. Lam, A. Qin, J. Liu, Z. Li and B. Z. Tang, Appl. Phys. Lett., 2007, 91, 011111; (b) H. Zhang, H. Li, J. Wang, J. Z. Sun, A. Qin and B. Z. Tang, J. Mater. Chem. C, 2015, 3, 5162; (c) H. T. Feng, S. Song, Y. C. Chen, C. H. Shen and Y. S. Zheng, J. Mater. Chem. C, 2014, 2, 2353; (d) X. Liu, J. Jiao, X. Jiang, J. Li, Y. Cheng and C. Zhu, J. Mater. Chem. C, 2013, 1, 4713; (e) Z. Zhao, J. W. Y. Lam and B. Z. Tang, J. Mater. Chem., 2012, 22, 23726; $(f)$ J. Huang, N. Sun, Y. Dong, R. Tang, P. Lu, P. Cai, Q. Li, D. Ma, J. Qin and Z. Li, Adv. Funct. Mater., 2013, 23, 2329; (g) X. G. Gu, J. J. Yao, G. X. Zhang, C. Zhang, Y. L. Yan, Y. S. Zhao and D. Q. Zhang, Chem. - Asian J., 2013, 8, 2362; (h) X. Zhang, Z. Chi, X. Zhou, S. Liu, Y. Zhang and J. Xu, J. Phys. Chem. C, 2012, 116, 23629; (i) J. Ma, T. T. Lin, X. Y. Pan and W. Z. Wang, Chem. Mater., 2014, 26, 4221; ( $j$ ) R. Misra, T. Jadhav, B. Dhokale and S. M. Mobin, Chem. Commun., 2014, 50, 9067; (k) K. R. Ghosh, S. K. Saha and Z. Y. Wang, Polym. Chem., 2014, 5, 5638; (l) W. Dong, T. Fei, A. Palma-Cando and U. Scherf, Polym. Chem., 2014, 5, 4048; $(m)$ J. Li, Y. Li, C. Y. K. Chan, R. T. K. Kwok, H. Li, P. Zrazhevskiy, X. Gao, J. Z. Sun, A. Qin and B. Z. Tang, Angew. Chem., Int. Ed., 2014, 53, 13518; (n) Z. Chang, Y. Jiang, B. He, J. Chen, Z. Yang, P. Lu, H. S. Kwok, Z. Zhao, H. Qiu and B. Z. Tang, Chem. Commun., 2013, 49, 594.

8 (a) J. He, B. Xu, F. Chen, H. Xia, K. Li, L. Ye and W. Tian, J. Phys. Chem. C, 2009, 113, 9892; (b) X. Q. Zhang, Z. G. Chi, B. J. Xu, L. Jiang, X. Zhou, Y. Zhang, S. W. Liu and J. R. Xu, Chem. Commun., 2012, 48, 10895; (c) H. Li, X. Zhang, Z. Chi, B. Xu, W. Zhou, S. Liu, Y. Zhang and J. Xu, Org. Lett., 2011, 13, 556; (d) L. Bu, M. Sun, D. Zhang, W. Liu, Y. Wang, M. Zheng, S. Xue and W. Yang, J. Mater. Chem. C, 2013, 1, 2028; (e) N. Song, D. X. Chen, M. C. Xia, X. L. Qiu, K. Ma, B. Xu, W. Tian and Y. W. Yang, Chem. Commun., 2015, 51, 5526; $(f)$ B. Xu, J. B. Zhang, S. Q. Ma, J. L. Chen, Y. J. Dong and W. J. Tian, Prog. Chem., 2013, 25, 1079.
9 (a) For reviews, see: J. W. Chen, B. Xu, X. Y. Ouyang, B. Z. Tang and Y. Cao, J. Phys. Chem. A, 2004, 108, 7522(b) Y. Ezhumalai, T. H. Wang and H. F. Hsu, Org. Lett., 2015, 17, 536; (c) T. Han, Y. J. Zhang, X. Feng, Z. G. Lin, B. Tong, J. B. Shi, J. G. Zhi and Y. P. Dong, Chem. Commun., 2013, 49, 7049; (d) Y. X. Guo, X. Feng, T. Y. Han, S. Wang, Z. G. Lin, Y. P. Dong and B. Wang, J. Am. Chem. Soc., 2014, 136, 15485.

10 (a) Y. Hong, J. W. Y. Lam and B. Z. Tang, Chem. Commun., 2009, 4332; (b) Y. Hong, J. W. Y. Lam and B. Z. Tang, Chem. Soc. Rev., 2011, 40, 5361; (c) A. J. Qin, J. W. Y. Lam and B. Z. Tang, Prog. Polym. Sci., 2012, 37, 182; (d) M. Wang, G. Zhang, D. Zhang, D. Zhu and B. Z. Tang, J. Mater. Chem., 2010, 20, 1858; (e) Z. Chi, X. Zhang, B. Xu, X. Zhou, C. Ma, Y. Zhang, S. Liu and J. Xu, Chem. Soc. Rev., 2012, 41, 3878; $(f)$ Z. Zhao, J. W. Y. Lam and B. Z. Tang, J. Mater. Chem., 2012, 22, 23726; (g) J. Mei, Y. N. Hong, J. W. Y. Lam, A. J. Qin, Y. H. Tang and B. Z. Tang, Adv. Mater., 2014, 26, 5429; (h) R. T. Kwok, C. W. T. Leung, J. W. Y. Lam and B. Z. Tang, Chem. Soc. Rev., 2015, 44, 4228; (i) H. Wang, E. Zhao, J. W. Y. Lam and B. Z. Tang, Mater. Today, 2015, 18, 365; (j) M. Chen, J. Z. Sun, A. Qin and B. Z. Tang, Chin. Sci. Bull., 2015, DOI: 10.1360/N972015-00760; (k) Z. Li and A. Qin, National Sci. Rev., 2014, 1, 22.

11 M. Chen, L. Li, H. Nie, J. Tong, L. Yan, B. Xu, J. Z. Sun, W. Tian, Z. Zhao, A. Qin and B. Z. Tang, Chem. Sci., 2015, 6, 1932.

12 (a) C. W. Ko, Y. T. Tao, A. Danel, L. Krzemińska and P. Tomasik, Chem. Mater., 2001, 13, 2441; (b) H. F. Yang, W. F. Su and Y. Chen, New J. Chem., 2011, 35, 1219; (c) E. Bellmann, G. E. Jabbour, R. H. Grubbs and N. Peyghambarian, Chem. Mater., 2000, 12, 1349; (d) Q. Y. Chen, L. Kong, Y. P. Tian, X. Y. Xu, L. M. Yang, G. B. Zhang, W. B. Jia and J. X. Yang, RSC Adv., 2014, 4, 18981.

13 (a) W. Z. Yuan, P. Lu, S. Chen, J. W. Y. Lam, Z. Wang, Y. Liu, H. S. Kwok, Y. Ma and B. Z. Tang, Adv. Mater., 2010, 22, 2159; (b) Z. Zhao, S. Chen, J. W. Y. Lam, P. Lu, Y. Zhong, K. S. Wong, H. S. Kwok and B. Z. Tang, Chem. Commun., 2010, 46, 2221.

14 (a) W. Qin, D. Ding, J. Liu, W. Z. Yuan, Y. Hu, B. Liu and B. Z. Tang, Adv. Funct. Mater., 2012, 22, 771; (b) W. Qin, K. Li, G. Feng, M. Li, Z. Yang, B. Liu and B. Z. Tang, Adv. Funct. Mater., 2014, 24, 635; (c) W. Huang, F. Tang, B. Li, J. Su and H. Tian, J. Mater. Chem. C, 2014, 2, 1141; (d) W. Huang, H. Wang, L. Sun, B. Li, J. Su and H. Tian, J. Mater. Chem. C, 2014, 2, 6834.

15 S. Cacchi, G. Fabrizi, A. Goggiamani, A. Iazzetti and R. Verdiglione, Synthesis, 2013, 1701.

16 H. Ren, J. Li, T. Zhang, R. Wang, Z. Gao and D. Liu, Dyes Pigm., 2011, 91, 298.

17 N. S. Nandurkar, M. J. Bhanushali, M. D. Bhor and B. M. Bhanage, Tetrahedron Lett., 2007, 48, 6573.

18 Y. Liu, S. M. Chen, J. W. Y. Lam, P. Lu, R. T. K. Kwok, F. Mahtab, H. S. Kwok and B. Z. Tang, Chem. Mater., 2011, 23, 2536.

19 (a) R. Hu, E. Lager, A. Aguilar-Aguilar, J. Liu, J. W. Y. Lam, H. H. Y. Sung, I. D. Williams, Y. Zhong, K. S. Wong, 
E. Peña-Cabrera and B. Z. Tang, J. Phys. Chem. C, 2009, 113, 15845; (b) E. Wang, J. W. Y. Lam, R. Hu, C. Zhang, Y. S. Zhao and B. Z. Tang, J. Mater. Chem. C, 2014, 2, 1801; (c) M. Chen, L. Li, H. Nie, Y. Shi, J. Mei, J. Wang, J. Z. Sun, A. Qin and B. Z. Tang, Chem. Commun., 2015, 51, 10710.

20 (a) X. Luo, J. Li, C. Li, L. Heng, Y. Q. Dong, Z. Liu, Z. Bo and B. Z. Tang, Adv. Mater., 2011, 23, 3261; (b) Z. Chen, J. Zhang, M. Song, J. Yin, G. A. Yu and S. H. Liu, Chem. Commun., 2015, 51, 326.
21 (a) L. Qian, B. Tong, J. Shen, J. Shi, J. Zhi, Y. Q. Dong, F. Yang, Y. P. Dong, J. W. Y. Lam, Y. Liu and B. Z. Tang, J. Phys. Chem. B, 2009, 113, 9098; (b) Y. Dong, J. W. Y. Lam, A. Qin, Z. Li, J. Sun, H. H. Y. Sung, I. D. Williams and B. Z. Tang, Chem. Commun., 2007, 40.

22 A. Qin, J. W. Y. Lam, H. Dong, W. Lu, C. K. W. Jim, Y. Dong, M. Häussler, H. H. Y. Sung, I. D. Williams, G. K. L. Wong and B. Z. Tang, Macromolecules, 2007, 40, 4879.

23 N. S. Makarov, M. Drobizhev and A. Rebane, Opt. Express, 2008, 16, 4029. 